\title{
Bioanalysis
}

\section{Clinical and Pharmaceutical Solutions through Analysis (CPSA BRASIL 2015): on the way to innovation - pharmaceutical/ analytical technology, regulation and knowledge management}

\section{Clinical and Pharmaceutical Solutions through Analysis, São Paulo, Brazil, 3-5 August 2015}

The 2nd Annual Symposium on Clinical and Pharmaceutical Solutions through Analysis was held on 3-5 August 2015 at Club Transatlântico, São Paulo, Brazil. This annual meeting began in 2014 and was the first industry-led event in Brazil to focus on the specific needs of industry researchers while bringing together technology and regulators. The goal of CPSA is to provide an in-depth review of innovative technology and industry practices through open discussion of industry-related issues and needs. Education and specialized training are the foundation of all CPSA events. As the industry has evolved so has CPSA. The CPSA annual meeting thrived with high quality scientific content, open interaction from industry opinion leaders and a collegial environment.

First draft submitted: 30 September 2015; Accepted for publication: 5 October 2015; Published online: 30 November 2015

Keywords: ANVISA • CPSA • pharmaceutical

Rafael Barrientos of Eurofarma served as the Program Chair for CPSA BRASIL 2015. This year's annual meeting turned-out to be another memorable event. Over the course of the meeting a theme developed in step with its title of using analytical and pharmaceutical technologies to innovate in step with regulations. The passion of the culture was evident as Brazil is focused on developing models for drug discovery and drug development.

Two preconference educational workshops were featured at CPSA BRASIL 2015. Daniel Lebre of CEMSA organized the workshop on 'Growing a Scientific Business.' It was wonderful to capture the enthusiasm of entrepreneurs in Brazil and to understand that the business model concepts are the same globally! Topics such as 'Scientific Business Development,' 'Scientific Business Strategy,' 'Patent Law in Brazil' and 'Marketing and Communication Plan' were presented by industry leaders. The other workshop was organized by SCIEX and hosted by Sindusfarma and included topics such as 'Introduction to Mass Spectrometry,' 'New Challenges by Pharma on Product Analysis' and 'Regulatory Affairs Overview.' Both workshops were interactive and led to many significant discussions throughout the preconference event and during the symposia sessions.

Rafael Barrientos and Mike Lee formally opened the CPSA BRASIL 2015 meeting on Tuesday with the introduction of the Plenary Lecturer, Nathan Yates, Professor at University of Pittsburgh. The lecture titled, "Differential Mass Spectrometry - Proteomics Applications in Basic, Translational, and Clinical Research" highlighted the role of mass spectrometry in basic research all the way to how the technology is influencing patients directly to make better decisions with their healthcare provider. Nathan's lecture also highlighted recent developments where his lab will be doing proteomics with a
Shane Needham ${ }^{*}, 1$ Nathan Yates $^{2}$, Rafael Barrientos ${ }^{3}$, Martin Steel ${ }^{4}$ \& Mike Lee ${ }^{5}$ 'Alturas Analytics, Inc. Moscow, ID, USA ${ }^{2}$ University of Pittsburgh, Pittsburgh, PA, USA

${ }^{3}$ Eurofarma, São Paulo, Brazil

${ }^{4}$ McKinley Scientific, Sparta, NJ, USA ${ }^{5}$ Milestone Development Services, Newtown, PA, USA

*Author for correspondence:

Tel.: +1 2088833400

sneedham@alturasanalytics.com

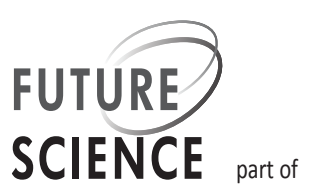


major sports franchise to determine how best to train, feed, rest and care for these athletes by testing individual biomarkers. This truly is 'personalized medicine' and will translate to the global population as the technology develops.

The majority of sessions on Tuesday and Wednesday concluded with a panel discussion where the speakers and moderator of the session took questions and comments from the audience. This was the most important and informative portion from each session. The discussions were engaging, educational and the passion for Brazil to learn and perform drug discovery and drug development was truly inspirational.

Tuesday's First Session Fixed Drug Combination (FDC): BA/BE, Pharmaceutical Equivalence and Regulatory Updates was chaired by Mauricio Magalhães, EMS and provided presentations by regulators from ANVISA (Gustavo Santos and Marcelo Angelo De Costa) and CRO's (Leonardo Teixeira, ICF). Most important were the lively discussions on the topics of how regulators and pharma can work together to develop better pharmaceuticals for the population. It is wonderful to see the complementary interactions of the regulators and industry in Brazil. Discussions also focused on how Brazil is transitioning away from an exclusive portfolio of $\mathrm{BE}$ studies for generic medicines, passing through FDC and to the drug discovery and development of novel pharmaceuticals. CROs, regulators and industry discussed in depth on regulatory issues and study design for BA/BE studies.

Dave Abramowitz of SCIEX presented a sponsored lunch talk and roundtable on the important topic of "Validation of LC/MS Instruments for Part 11 Compliance - How To Migrate to An Integrated Compliance Strategy for Your Lab." Based on the questions and interest from the audience and as guidance develops in Brazil, compliance of instruments will be a major component of success in LC-MS regulated labs.

The first afternoon session on Tuesday was chaired by Carlos Kiffer of GC2 (São Paulo, Brazil) - "Clinical Trials in Brazil: Regulatory Updates and Challenges." The pharma and CRO's were represented with discussions focused on the importance of meeting deadlines and transparency. The speakers also generated a good debate on the revised Brazilian regulation for clinical assays, which were released at the end of 2014. The final session on Tuesday afternoon was New Drug Release and Pharmaceutical Process Technologies: An Overview and was chaired by Gabriel Araújo of University of São Paulo (São Paulo, Brazil). Industry (Miller Nunes de Freitas, Aché Laboratórios Farmacêuticos), academia (Gabriel Araújo, University of São Paulo) and regulators (Natália Bellan, Inteligência Sanitária) were represented and the discussion was lively.
The CPSA BRASIL 2015 poster session was a highlight of the afternoon. The poster session was preceded by the first inaugural ' 5 Minutes of Fame' Vendor Session at CPSA BRASIL. The event was robust and spirited with new product introductions and services highlighting the vendor presentations. This unique session allows all CPSA vendors to showcase their products and services - in $5 \mathrm{~min}$ ! The energy and camaraderie demonstrated by the Brazilian vendors was amazing especially for the inaugural event as this same energy took 4-5 years to happen at the ' 5 Minutes of Fame' in the USA.

The Wednesday morning plenary lecture was delivered by Shane Needham of Alturas Analytics, Inc. (Moscow, Idaho) and was titled, "Analytical Technology: From the Analytical Chemistry Bench Straight Results to Patients: Is This Really True?”. The lecture highlighted the history, current status and future of handheld devices to measure biomarkers real time by patients. Most exciting with the discussion was the role that analytical chemists play in developing these instruments and diagnostic tests. In line with the plenary lecture from Professor Yates on Tuesday, Shane's lecture highlighted the future of personalized medicine through miniature instruments and customized diagnostic tests performed by the patient.

The morning session on Wednesday centered on "Biosimilars: where are we and where are we going?" and was chaired by Vanda Magalhaes, Eurofarma (São Paulo, Brazil). The session involved speakers from industry and regulatory agencies. Biosimilars are a growing trend in Brazil as the country has already demonstrated they can develop generic drugs, the rational next step was biosimilars. This trend has created an optimism in Brazil to perform drug discovery and development with large molecules. Marcelo Moreira of ANVISA (Brasília, Brazil) updated the audience on "present Brazilian regulatory scenario and trends." Two speakers from industry discussed the tactics of biosimilars with presentations on "how far should a comparability exercise goes?" Priscila Scheinberg, of Orygen São Paulo, Brazil and "are switching studies necessary for interchangeability?” Maritê Faria of Amgen in São Paulo, Brazil. Both presentations gave the audience a chance to contemplate critical elements about biosimilar development.

The Wednesday sponsored lunch and roundtable discussion was sponsored by Agilent Technologies where José Xavier discussed the innovative LC-MS solutions for biopharma. Later in the lunch event, Gabriela Venturin of the University of São Paulo discussed Metabolomic studies in a Brazilian population - Use of GC/MS for Metabolite Phenotype Characterization. 
The Wednesday afternoon symposia session was appropriately titled, "Drug discovery: are we prepared for that?" and was Chaired by Gabriela Barreiro of Eurofarma. This session was timely and lively as it summarized a common discussion point at the conference about doing drug discovery in Brazil. Presentations were given from industry and academia. Presentations included, "Advances in natural-product-based screening from Brazilian Biomes" by Cristina Dislich Ropke of Phytobios (São Paulo, Brazil), "The role of academia in drug discovery" by Lídia Moreira Lima, UFRJ (Rio de Janeiro, Brazil) and "The role of information technology in the development of new drugs Cristina Mendes, INPI (Brasília, Brazil). The discussion of this session was focused on how Brazil could be inserted into this entirely new world for the Brazilian Pharmaceutical Industry. Governmental policies for innovation, knowledge import for improving the learning curve and cooperation between academy and industry are key for this purpose.

Wednesday evening closed with a session about, "Personalize medicine with better diagnoses, better treatment through innovative analytical methods" chaired by Daniel Lebre of CEMSA (São Paulo, Brazil) and Dulce Casarini, UNIFESP (São Paulo, Brazil), Daniel Lebre discussed "therapeutic imunossupressor monitoring using dried blood spot and LC-MS/MS" Dulce Casarini discussed "clinical biomarkers" and Valdemir Melechco Carvalho of Fleury (São Paulo, Brazil) discussed "application of directed proteomics in clinical biochemistry."
The closing event of the conference was sponsored by CEMSA and Peak Scientific. Of course no Brazilian event is complete without soccer and Brazilian style barbecue! Our game and festivities went well into the night and discussions focused on friends, family and science.

Plans are already underway for next year's annual meeting, the 3rd Annual Symposium on Clinical and Pharmaceutical Solutions through Analysis (CPSA BRASIL 2016). The theme for CPSA BRASIL 2016 is "Pharmaceutical Innovation: Collaborating from Bench to Bedside" and will be held on August 1-3, 2016 at the Club Transatlântico in São Paulo, Brazil. Carlos Kiffer of GC2 (São Paulo, Brazil) will serve as Program Chair. For more information and details regarding CPSA BRASIL 2016, please visit [1].

\section{Financial \& competing interests disclosure}

The authors have no relevant affiliations or financial involvement with any organization or entity with a financial interest in or financial conflict with the subject matter or materials discussed in the manuscript. This includes employment, consultancies, honoraria, stock ownership or options, expert testimony, grants or patents received or pending, or royalties.

No writing assistance was utilized in the production of this manuscript.

\section{Reference}

1 CPSA BRASIL 2016. www.cpsa-brasil.com 